\title{
IN VITRO SELECTION AND BINDING STUDIES OF A DNA APTAMER TARGETING PHOSPHATIDYLSERINE (PS) USING NON-FLUORESCENCE MEASUREMENTS
}

\author{
HAMDY M. EMBARK \\ Animal Physiology Department, Faculty of Vet. Medicine, South Valley University, Qena 83523, Egypt
}

Received: 10 October 2016; Accepted: 31 October 2016

\begin{abstract}
Phosphatidylserine (PS) is a phospholipid that is an important component of all cell membranes. Normally, phospholipid PS is only found on the cytosolic surface of the plasma membrane. Obligatory translocation of PS from the interior to the exterior side of the plasma membranes is a potential biomarker for apoptotic cell death. The SELEX technology (Systematic Evolution of Ligands by Exponential enrichment) is widely applied as an in vitro selection and amplification method to generate target-specific aptamers. In this study, entropic fragment based approach (EFBA) was used to design a set of DNA aptamers bind specifically with PS based on seed-andgrow strategy. Each member of designed short-sequence DNA aptamers consists of a 6 nucleotides while longsequence DNA aptamers consists of an 11 nucleotides. The binding properties of the designed DNA aptamers with phospholipid PS have been studied using NanoDrop without fluorescent tags attached to them. This technique has been proposed to measure concentrations of relative PS bound aptamers versus unbound ones and to analyze the phospholipid binding properties of aptamers. The Short-sequence DNA aptamers show stronger binding affinity for phospholipid PS compared with long ones. Also short-sequence DNA aptamer (AAAGAC) has the highest binding affinity compared with the other short ones and also the other two long ones. These results suggest that the phospholipid PS binding of DNA aptamers is very much sequence specific and depends also on the number of nucleotides in the DNA aptamers. The present results thus disclose a novel assay for phospholipid PS recognition in apoptotic cells, which may open up the possibility of discovering aptamer based diagnostic tools to be used in treatment of many diseases including cancer.
\end{abstract}

Key words: Phosphatidylserine, DNA Aptamers, Apoptosis, Cancer

\section{INTRODUCTION}

Phospholipids are actually a very important component of cell membranes that provide structure, function, and protection to cells (Dowhan and Bogdanov, 2002). The major lipid components structures of the eukaryotic cell membranes are namely phosphatidylethanolamine (PE), phosphatidylcholine (PC), phosphatidylserine (PS), phosphatidylinositol (PI), and phosphatidic acid (PA) (Chaurio et al., 2009). The glycerophos pholipidsphos phatidylcholine (PC) is the main phospholipid component (comprising more than $50 \%$ of the phospholipids) in most eukaryotic cell membranes and functions as a neutral type lipid which is responsible for creating membrane planar bilayers (Van Meer et al., 2008). While, PS is present at low levels(less than 10\%) in the plasma membrane of normal eukaryotic cells and plays a key role in cell cycle signaling, specifically in relationship to apoptosis. (Mariño and Kroemer, 2013).
In general terms, the outer membrane leaflet of the plasma membrane of normal eukaryotic cells is formed predominantly with Phospholipids PC and PE, whereas the majority of the Phospholipids PS, $\mathrm{PE}$, and PI are confined to the membrane's inner leaflet (Zwaal, and Schroit, 1997; Fadeel and Xue, 2009) (Fig. 1). The redistribution of PS between internal and external leaflet of the plasma membranes is known as PS externalization (Kagan et al., 2000) and has been recently shown to be an important marker of the induction of programmed cell death (apoptosis) (Lee et al., 2013). Apoptosis is considered a vital, highly regulated, and natural component of various processes (Elmore, 2007) that contributes to the normal development and maintenance of human and animal cells extending from embryonic development to the maintenance of normal cell number homeostasis (Weinberg, 2013). 


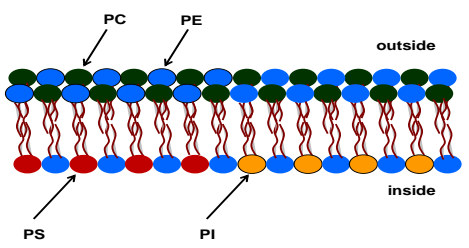

Fig. 1: Distribution of phospholipids in the plasma membrane of normal eukaryotic cells. The major phospholipids are phosphatidylethanolamine (PE), phosphatidylcholine (PC), phosphatidylserine (PS), and phosphatidylinositol (PI).

The phospholipid PS externalization on the surface of apoptotic cell membranes is an attractive target for the detection of apoptosis (Blankenberg, 2009) which can be used to provide an early indication of the success or failure of cancer chemotherapy (Yang et $a l ., 2015)$. A number of Molecular probes have been developed to detect PS externalization in apoptotic cells. Annexin V (annexin A5), a naturally occurring $36 \mathrm{kDa}$ human phosphatidyl binding protein, has been most extensively investigated (Blankenberg, 2008). However, the annexins have exhibited a variety of disadvantages as noninvasive imaging probes of apoptosis, as detailed by Boersma et al. (2005), including high uptake in normal tissues, especially liver and kidney, long biological half-life in nontarget tissues, high radiation burden for analogs and laborious radiochemistry for their labeling. Identification of alternative constructs free from these shortcomings will definitely provide vital imaging tools to detect apoptosis.

Over the past several years, antibodies and aptamers were developed to target either PC or PS as diagnostic agents or therapeutic inhibitors (Proske et $a l ., 2002)$. The therapeutic strategy is to inhibit either the conversion by blocking the associated hot spots on PC or the aggregation by binding directly to PS. However, the treatments using immunotherapy remain controversial (Campana et al., 2009). Because recent studies of prion disease in membrane environments show that the presence of PS alters amyloid aggregation pathways and increases aggregation (Thellung et al., 2011), it suggests an attractive alternative route, namely, targeting PS, for treatments. Furthermore, Chiesa et al. (2000) has shown intracellular PS aggregation in membranes triggers apoptosis. Consequently, monitoring the changes of PS externalization also could provide an early indication of the success or failure of therapy for prion diseases.

Oligonucleotide aptamers possess a number of desirable properties as potential drug entities being characterized by strong and specific binding to cellular targets, ease of synthesis, attractive pharmacokinetics and low toxicity (Nimjee et al., 2005; Pan and Clawson, 2009). These desirable properties have motivated us to design theranostic aptamers which will target PS. Oligonucleotide aptamers are traditionally identified through the process of Systematic Evolution of Ligands by EXponential enrichment (SELEX) (Yüce et al., 2013). Although the SELEX technology is well developed, several issues including the design of randomized DNA/RNA libraries and the complexity of the selection process still pose major challenges to its applications (Tseng et al., 2011).

Tseng et al. (2011) has started to explore a computational entropic fragment based approach (EFBA) for aptamer template design based solely on the knowledge of the biomolecular target structures. This approach may overcome some of the problems encountered in aptamer selection through SELEX. Basically, this approach integrates information processing methods with the seed-and-grow strategy to determine the probability distribution of the nucleotide sequences that most likely interact with target structures. Using this computational method, the nucleotide sequences and the corresponding tertiary structures are determined simultaneously. With the advancement of our knowledge of theoretical aptamer design and the properties of aptamers, PS targeting aptamers can be efficiently designed and readily tested. These PS targeting aptamers once designed by computational methods can be obtained by commercial custom synthesis and further studied in cell-based experimental assays and then optimized by iteractive redesign.

In the current study, a novel method has been developed to assess aptamer/lipid binding properties using liposome technology and NanoDrop spectrophotometer without fluorescence tags. Therefore, the binding behavior of computationally derived sequences from DNA aptamers can be tested and the best ones binding with PS are selected diretly. In addition, this study identify the best possible candidate for further modifications aimed at practical implementations as either an imaging tool in cell based studies or as a therapeutic agent.

\section{MATERIALS AND METHODS}

\section{Entropic fragment based approach (EFBA) to PS binding DNA aptamer design}

The foundation of the proposed approach lies in the answer to the following question: "Given the structural information of the target, what is the preferred probable distribution of having an aptamer that is most likely to interact with the target?" (Tseng et al., 2011). Once the aptamer problem is tackled by asking this question, the problem can be solved using information theory and seed-and-grow strategy. The proposed approach consists of three steps. First, it 
determines the probable distribution of the preferred first nucleotide (seed) based on the input information such as the total or interaction energy of the single nucleotide-target complex based on the maximum entropy method. Second, given the probable distribution of the preferred nucleotide obtained in the previous step, it determines the probable distribution of preferred neighboring nucleotides based on the input information using the maximum entropy method again. By repeating this same procedure, one can obtain the joint probable distribution of an L-mer nucleotide sequence $P_{\mathrm{L}-\mathrm{mer}}$ that is most likely to interact with the target. Third, it applies the entropic criterion defined by the relative entropy, $\mathrm{S}\left[P_{\mathrm{L}}\right.$ $\left.{ }_{\text {met }} \mid P_{\text {ref }}\right]=-\sum_{\mathrm{i}} P_{\mathrm{L}-m e t}(\mathrm{i}) \log P_{\mathrm{L}-m e r}(\mathrm{i}) / P_{\text {ref }}(\mathrm{i})$, where the reference probable $P_{\text {ref }}$ is set to be a uniform distribution, which represents our complete ignorance regarding the interaction to determine the preferred sequence and its length $L$ and $i$ labels conformations of the sequence. The decreased $\mathrm{S}\left[P_{\mathrm{L}-\text { met }} \mid P_{\text {ref }}\right]$ indicates the preference of $\log P_{\mathrm{L}-\mathrm{mer}}$. The reader canreferre to Tseng et al. (2011) for further details.

\section{In vitro binding studies}

\section{Liposomes preparation}

Liposome technology was used to assay the binding targets of the computationally-derived aptamers for its speed and simplicity (Hope et al., 1986). The phospholipid PS (1,2-dipalmitoyl-sn-glycero-3phospho-L-serine (sodium salt) (DPPS) in powder form) was obtained from Avanti Polar Lipids (Alabaster, AL, USA). For liposome preparation, the phospholipid was dissolved in Methanol $(\mathrm{MeOH})$ to prepare a stock solution $(1 \mathrm{mg} / \mathrm{ml})$ by dissolving $6.7 \mathrm{mg}$ DPPS or $6.7 \mathrm{mg}$ DPPC in $6.7 \mathrm{ml} \mathrm{MeOH}$.

\section{Non-fluorescence lipid-binding assay}

The detailed protocol published previously (Tseng et $a l ., 2011)$ is summarized as follows. Fifty microliter of the $\mathrm{MeOH}$ solution of the lipids was transferred to each Eppendorf tube and evaporated to dryness by incubation overnight followed by addition of appropriate volume of the test aptamer as a solution in Tris/EDTA buffer $(10 \mathrm{mM}$ stock or stock with further dilution) to ensure the desired experimental aptamer concentration in solution.

The lipid/aptamer mixture was incubated for 60 minutes in the dark. Supernatants (buffer and unbound aptamers) were then removed. Sixty microliter of Tris/EDTA buffer was added to the eppendorf tubes to resuspend the lipid followed by centrifugation and the supernatants were again removed. This process was repeated three times to effectively remove unbound aptamers. Required amount of $\mathrm{MeOH}$ was then added to each eppendorf tube containing bound aptamer/phospholipids to make the final volume to be $60 \mu \mathrm{L}$. Onemicroliter from this final suspension was loaded onto NanoDrop(Thermo Scientific NanoDrop 2000 spectrophotometer) and the DNAconcentration was measured at 260/280 $\mathrm{nm}$ for each sample.

\section{RESULTS}

\section{Aptamers designed based on the total energy}

Two pieces of input information factors, total and interaction energy of the aptamer-phospholipid complex were used in the design. Here the total energy was used first. The PS is target and since the lipid portion of many lipids is similar, the head group of PS has been used for the design as shown in the equilibrated PS structure generated using Visual Molecular Dynamics (VMD) (Humphrey et al., 1996) in Fig. 1.

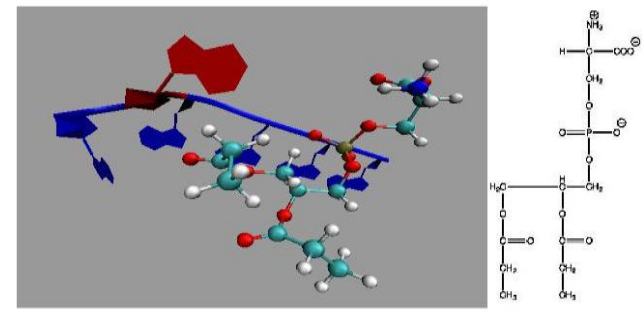

Fig. 1: Tertiary structure of AAA AGA-PS complex. This figure is taken with due permission from (Ashrafuzzaman et al., 2013). The tertiary structure of the Microchip electrophoresis (ME) aptamer (AAA AGA)-PS complex generated using VMD (Schmidt et $a l ., 1993$ ) and the two-dimensional structure of PS are shown. Note that this complex was used as one of initial structures for molecular dynamic simulation studies. Adenine is colored by blue and guanine is red.

The right-hand side shows its two-dimensional structure. Having determined the first nucleotide (seed), the rest of the sequence was determined in a similar fashion until the stopping criterion was met. It suggests that the nucleotides added after either the $5^{\text {th }}$ or $6^{\text {th }}$ step do not further enhance the interactions between the nucleotide fragment and PS. Therefore, the present study conclude that the 6-mer is the preferred length. The top four short sequences and top two longsequences aptamerslisted in Table.1 were chosen for this study. 
Table 1: DNA aptamers (short- and long-sequences) used Direct detection of bound short sequences aptamers with DPPS

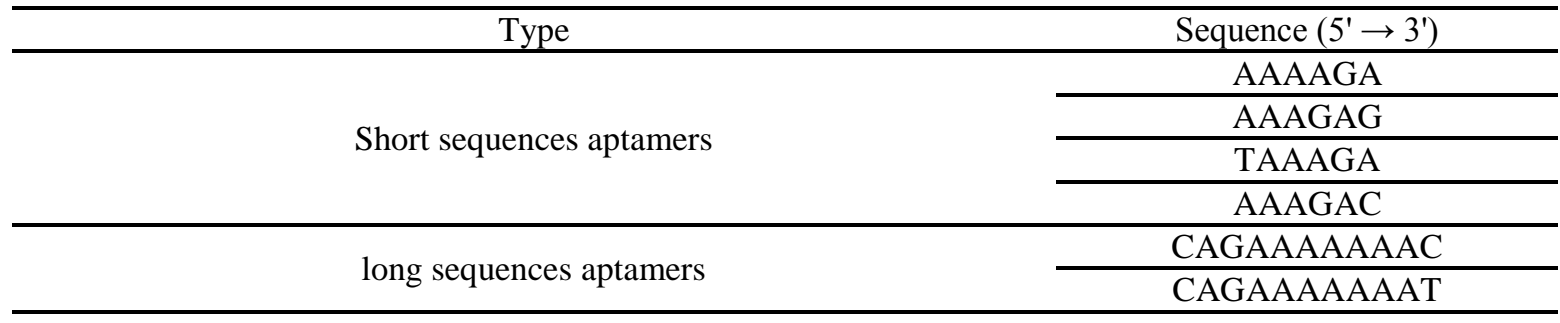

Short sequences aptamers show stronger binding affinity for PS. The liposomes prepared in this study were designed to mimic a non-binding target for PS targeting aptamers. Fig. 2 shows the non-fluorescence intensity from bound DNA aptamers of short sequences using various concentrations (3.33 to $30 \mu \mathrm{M})$ of DNA aptamers with the DPPS liposomes. It suggests that short sequences of DNA aptamers bind specifically to DPPS liposomes. Thus, this indicates that the binding of the aptamers is specific to the liposomes containing the serine head group. Although short sequences of DNA aptamers seem to bind to DPPS containing liposomes, in vitro results indicate AAA GAC had the highest binding level, followed by TAA AGA and AAA AGA respectively. These results indicate that short sequence aptamer (AAA GAC) tested in this study has high binding affinity for DPPS.

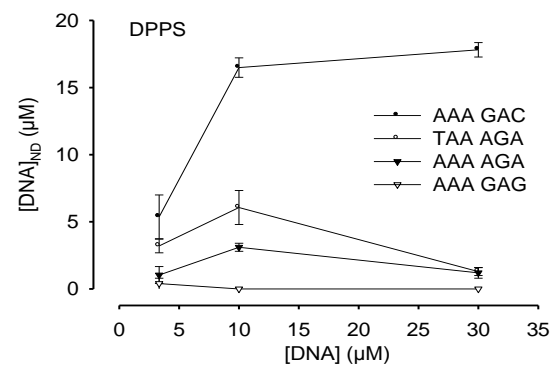

Fig. 2: Binding affinity and selectivity of designed short sequences DNA aptamers. Selective binding of the top four short sequences aptamers AAA GAC, TAA AGA, AAA AGA, and AAA GAG with liposomes containing PS.

\section{Direct detection of bound long sequences aptamers with DPPS}

Fig. 3 shows the non-fluorescence intensity from bound of DNA aptamers of long sequences DNA aptamers (CAGAAAAAAAC and CAGAAAAAAAT) using various concentrations (3.33 to $30 \mu \mathrm{M}$ ) of long sequences DNA aptamers with the DPPS liposomes. It suggests that long sequence of DNA aptamer (CAGAAAAAAAC) bind specifically to DPPS liposomes compared with the long sequence of DNA aptamer (CAGAAAAAAAT), which has no binding affinity to DPPS at all. Thus, this indicates that the binding of the aptamers is specific to the liposomes containing the serine head group with selectivity and specificity.

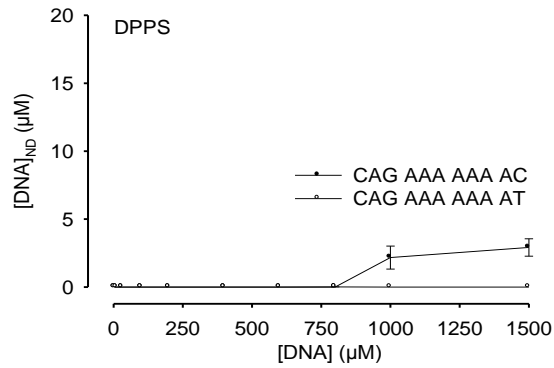

Fig. 3: Binding affinity and selectivity of designed long sequences DNA aptamers. Selective binding of the top two long sequences DNA aptamer CAGAAAAAAAC and CAGAAAAAAAT with liposomes containing PS.

\section{Direct detection of bound short sequence aptamer (AAA GAC) with DPPS Versus DPPC}

The binding affinity of the top short sequence DNA aptamer (AAA GAC) to DPPS is compared with its binding to liposomes containing PC. Fig. 4 shows the non-fluorescence intensity from bound DNA aptamers of short sequence (AAA GAC) using various concentrations $(3.33$ to $30 \mu \mathrm{M})$ of DNA aptamer (AAA GAC) with the DPPS and DPPC liposomes. It suggests that short sequence DNA aptamer (AAA GAC) binds specifically to DPPS liposomes with poor binding affinity for DPPC. Thus, this indicates that the binding of the aptamers is specific to the liposomes containing the serine head group.

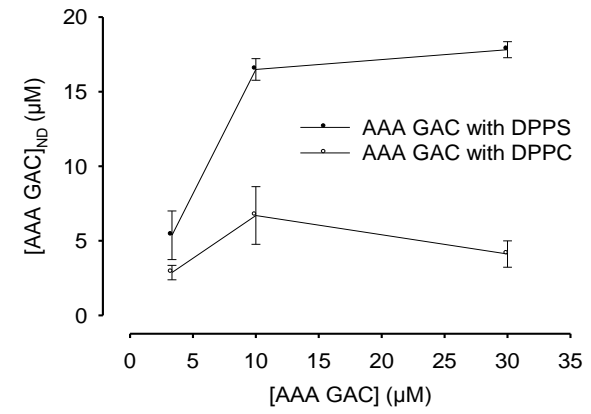

Fig. 4: Binding affinity and selectivity of designed short sequence DNA aptamer (AAA GAC). Selective binding of aptamer AAA GAC with liposomes containing PS and PC. 


\section{DISCUSSION}

Total and interaction energy of the aptamerphospholipid complex were used in the design of DNA aptamers according to a previous study (Tseng et al., 2011). Top four short sequences of DNA aptamers and top two long sequences DNA aptamers are used in this study as determined previously (Ashrafuzzaman et al., 2013) using indirect fluorescence detection method. In this study, a direct method using NanoDrop without fluorescence tags was used to compare between two studies and also will not expensive method for detection.

High binding affinity of short sequence DNA aptamers compared with long ones. Also short sequence DNA aptamer (AAA GAC) has the highest binding affinity compared with the other short one and also the other two long ones. These results indicate that the best aptarner bind with PS can be selected specifically. The target is PS for its clinical important application as it was shown that PS migrates to outside of the plasma membrane during apoptotic processes. For clinical use, we could use PS as biomarker to indicate induction of apoptosis especially during cancer treatment. If we succeed to select and characterize a specific aptamer which able to bind specifically with PS, so we could be able to diagnose and follow up the cancer treatment course.

\section{CONCLUSIONS}

An entropic fragment based approach (EFBA) has been applied to the design of a DNA aptamer which would bind specifically to PS. The In vitro experimental studies conducted here have provided a better understanding of the designed aptamers' properties beyond those uncovered in a previous study (Tseng et al., 2011). The results presented here show that the designed sequences bind selectively to the liposomes having a PS surface with varying affinity.

The current study show that AAA GAC can be utilized as a scaffold for the development of novel agents for either therapeutic or diagnostic purposes targeting PS in a cell membrane. As stated in the introduction, two potential applications of the improved AAA GAC-based aptamer can be considered although there are many others: (i) as an apoptotis detector which would replace the existing probes such as annexins, (ii) as a therapeutic drug for inhibiting amyloid aggregation in prion disease.

\section{ACKNOWLEDGEMENTS}

The author gratefully acknowledges all members and professors of the department of animal physiology, faculty of veterinary medicine, south valley university, Qena, Egypt, and of biochemistry department and Protein Research Chair, faculty of science, King Saud university, Riyadh, Saudi Arabia, for their constant encouragement and kind help.

\section{REFERENCES}

Ashrafuzzaman, M.; Tseng, CY.; Kapty, J.; Mercer, JR. and Tuszynski, JA. (2013): A computationally designed DNA aptamer template with specific binding to phosphatidylserine. Nucleic Acid Ther. 23(6): 418-426.

Blankenberg, F.G. (2008): In vivo imaging of apoptosis. Cancer Biology \& Therapy, 7(10): $1525-1532$.

Blankenberg, F.G. (2009): Imaging the molecular signatures of apoptosis and injury with radiolabeled annexin V. Proceedings of the American Thoracic Society, 6(5): 469-476.

Boersma, H.H.; Kietselaer, B.L.; Stolk, L.M.; Bennaghmouch, A.; Hofstra, L.; Narula, J.; Heidendal, G.A. and Reutelingsperger, C.P. (2005): Past, present, and future of annexin A5: from protein discovery to clinical applications. Journal of Nuclear Medicine, 46(12): 2035-2050.

Campana, V.; Zentilin, L.; Mirabile, I.; Kranjc, A.; Casanova, P.; Giacca, M.; Prusiner, S.B.; Legname, $G$. and Zurzolo, C. (2009): Development of antibody fragments for immunotherapy of prion diseases. Biochemical Journal, 418(3): 507-515.

Chaurio, R.A.; Janko, C.; Muñoz, L.E.; Frey, B.; Herrmann, M. and Gaipl, U.S. (2009): Lipids: key players in apoptosis and immune regulation. Molecules. 14(12): 4892-4914.

Chiesa, R.; Drisaldi, B.; Quaglio, E.; Migheli, A.; Piccardo, P.; Ghetti, B. and Harris, D.A. (2000): Accumulation of protease-resistant prion protein $(\mathrm{PrP})$ and apoptosis of cerebellar granule cells in transgenic mice expressing a PrPinsertional mutation. Proceedings of the National Academy of Sciences, 97(10): 55745579 .

Dowhan, W. and Bogdanov, M. (2002): Functional roles of lipids in membranes. In: Biochemistry of Lipids, Lipoproteins and Membranes, $4^{\text {th }}$ ed.; Vance, D.E., and Vance, J.E., (eds), Elsevier Science B.V., Amsterdam. pp 1-35.

Elmore, S. (2007): Apoptosis: a review of programmed cell death. Toxicol. Pathol. 35(4): 495-516.

Fadeel, B. and Xue, D. (2009): The ins and outs of phospholipid asymmetry in the plasma membrane: roles in health and disease. Crit. Rev. Biochem. Mol. Biol. 44(5): 264-277.

Humphrey, W.; Dalke, A. and Schulten, K. (1996): VMD: Visual molecular dynamics. Journal of Molecular Graphics, 14(1): 33-38.

Kagan, V.E.; Fabisiak, J.P.; Shvedova, A.A.; Tyurina, Y.Y.; Tyurin, V.A.; Schor, N.F. and Kawai, K. 
(2000): Oxidative signaling pathway for externalization of plasma membrane phosphatidylserine during apoptosis. FEBS Lett. 477(1-2): 1-7.

Lee, S.H.; Meng, X.W.; Flatten, K.S.; Loegering, D.A. and Kaufmann, S.H. (2013): Phosphatidylserine exposure during apoptosis reflects bidirectional trafficking between plasma membrane and cytoplasm. Cell Death Differ. 20(1): 64-76.

Mariño, G. and Kroemer, G. (2013): Mechanisms of apoptotic phosphatidylserine exposure. Cell Res. 23(11): 1247-1248.

Nimjee, S.M.; Rusconi, C.P. and Sullenger, B.A. (2005): Aptamers: An emerging class of therapeutics. Annual Review of Medicine, 56: 555-583.

Pan, W. and Clawson., GA. (2009): The shorter the better: reducing fixed primer regions of oligonucleotide libraries for aptamer selection. Molecules. 14(4): 1353-1369.

Proske, D.; Gilch, S.; Wopfner, F.; Schätzl, H.M.; Winnacker, E.L. and Famulok, M. (2002): Prion-protein-specific aptamer reduces PrPSc formation. ChemBioChem., 3(8): 717-725.

Schmidt, M.W.; Baldridge, K.K.; Boatz, J.A.; Elbert, S.T.; Gordon, M.S.; Jensen, J.H.; Koseki, S.; Matsunaga, N.; Nguyen, K.A.; Su, S.; Windus, T.L.; Dupuis, M. and Montgomery, J.A. (1993): General atomic and molecular electronic structure system. Journal of Computational Chemistry, 14(11): 1347-363.
Thellung, S.; Corsaro, A.; Villa, V.; Simi, A.; Vella, S.; Pagano, A. and Florio, T. (2011): Human PrP90-231-induced cell death is associated with intracellular accumulation of insoluble and protease-resistant macroaggregates and lysosomal dysfunction. Cell Death and Disease, 2: e138.

Tseng, C.Y.; Ashrafuzzaman, M.; Mane, J.Y.; Kapty, J.; Mercer, J.R. and Tuszynski, J.A. (2011): Entropic fragment based approach to aptamer design. Chem. Biol. Drug Des. 78, 1-13.

Van Meer, G.; Voelker, D.R. and Feigenson, G.W. (2008): Membrane lipids: where they are and how they behave. Nature Reviews Molecular Cell Biology, 9(2): 112-124.

Weinberg, R.A. (2013): The biology of Cancer. $2^{\text {nd }}$ Ed. Garlan Science, New York, USA. pp: 357.

Yang, M.; Jiang, P. and Hoffman, R.M. (2015): Early Reporting of Apoptosis by Real-time Imaging of Cancer Cells Labeled with Green Fluorescent Protein in the Nucleus and Red Fluorescent Protein in the Cytoplasm. Anticancer Res. 5(5): 2539-2543.

Yüce, M.; Ullah, N. and Budak, H. (2015): Trends in aptamer selection methods and applications. Analyst. 140(16): 5379-5399.

Zwaal, R.F. and Schroit, A.J. (1997): Pathophysiologic implications of membrane phospholipid asymmetry in blood cells. Blood. 89(4): 1121-1132.

\section{انتقاء مَعْمَليّ ودراسات تر ابطية لأبتمر الحمض النووي الصبغي (DNA) المستهدف لفوسفاتيديل

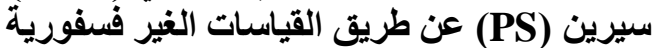 \\ حدبي أمبارك}

E-mail: h.embark@vet.svu.edu.eg; $\underline{\text { atutohm@yahoo.com Assiut University web-site: www.aun.edu.eg }}$

فسفاتيديل سيرين (PS) عبارة عن نوع من الدهن الفوسفوري أو الليبيدات المفسفرة (فوسفوليييد) و التي تعد مكوّناً أساسياً من الغشاء

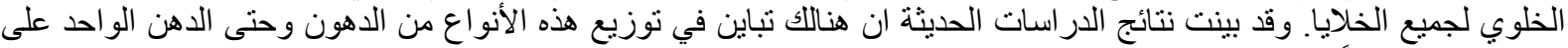

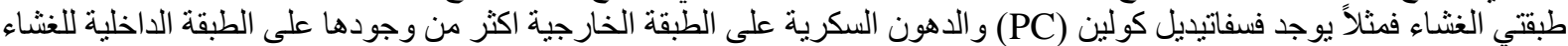

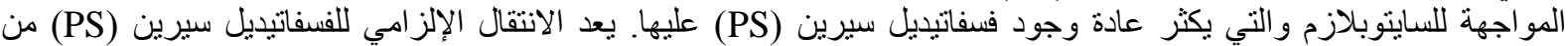

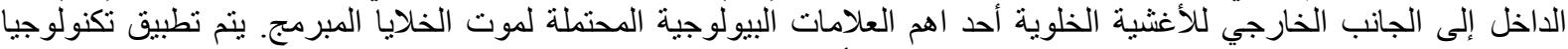

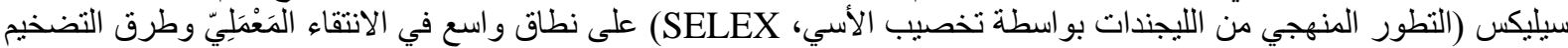

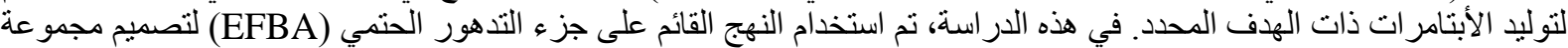

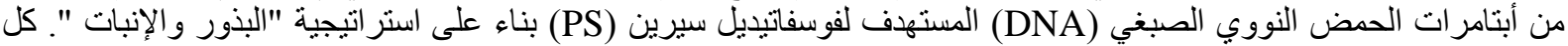

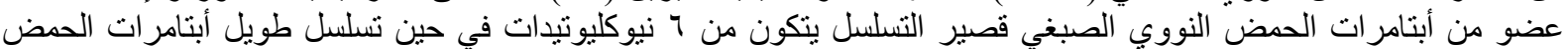

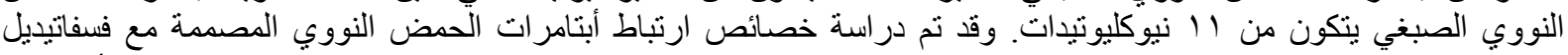

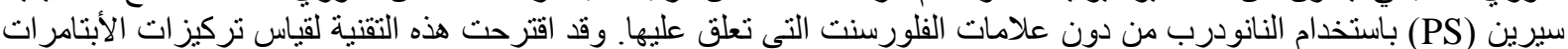

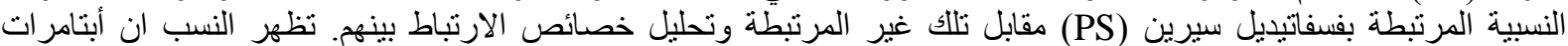

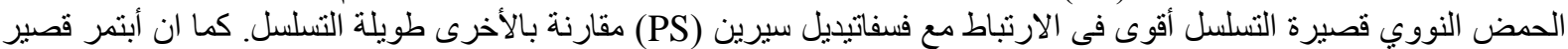

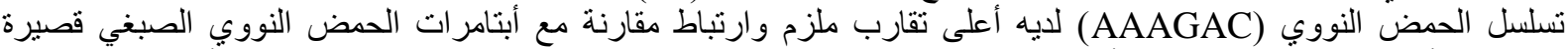

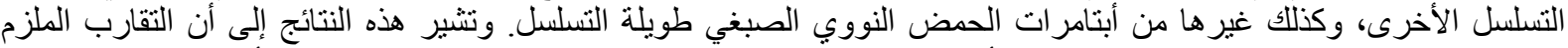

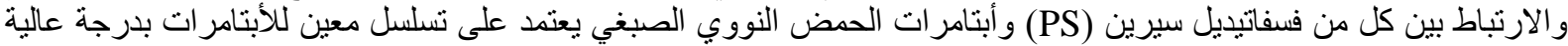

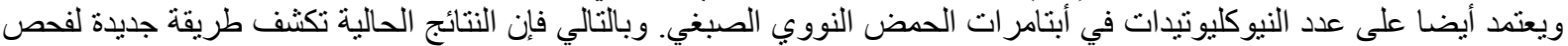

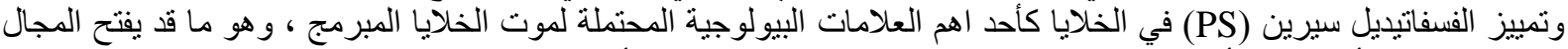
لإمكانية اكتثاف أبتامر ات كأدو ات للتشخيص واستخدامها في علاج العديد من الأمر اض بما فيها السرطان. 